\title{
LONGITUDINAL ANALYSIS OF ANTIMICROBIAL CONSUMPTION IN NEONATAL INTENSIVE CARE UNIT: THE USE OF DEFINED DAILY DOSES
}

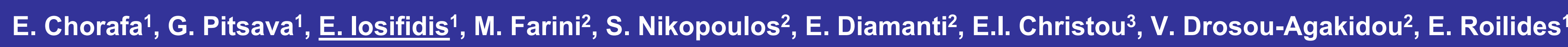

${ }_{1}^{1} 3^{\text {rd }}$ Pediatric Department, Aristotle University and Hippokration Hospital, Thessaloniki, Greece

$21^{\text {st }}$ Neonatal Department and Neonatal Intensive Care Unit, Aristotle University of Thessaloniki, Thessaloniki, Greece 3Pharmacy department, Hippokration Hospital, Thessaloniki, Greece

\section{Background}

Monitoring of antimicrobial consumption in neonates is essential, but there are limited longitudinal data because of the absence of standardized metrics. ${ }^{1,2}$

Defined daily doses (DDDs) are recommended by the World Health Organization for monitoring antimicrobial consumption currently only for adults. ${ }^{3}$

\section{Aim}

To use the methodology of DDDs and assess the pattern and time trends of antibiotic consumption (AMC) in a Neonatal Intensive Care Unit (NICU) for 15 years.

\section{Methods}

Study design: Retrospective study

Setting: A 44-bed neonatal unit (15 NICU beds) in a tertiary-care level hospital in Thessaloniki, Greece

Duration: January 2002 to December 2016

Time periods: 2002-2006, 2007-2011, 2012-2106

Data source: Data of AMC were obtained from the hospital pharmacy and number of bed-days was obtained from Hospital Office of Statistics.

Measure of antimicrobial use: AMC was expressed as defined daily doses per 100 bed-days (DDDs/100BD), as recommended by the World Health Organization for adults ${ }^{1}$. The software used for the data processing was the ABC Calc (Microsoft $\circledast)^{4}$.

N DDDs of an antibiotic $=\frac{\text { grams of antibiotic consumed }}{\text { Gram per DDD(WHO)) }}$

Only antimicrobial agents (antimicrobial J01) used for systematic use were recorded.

Results

The median total AMC ranged between 21.2 and 24.8DDD/100BD during study period.(Figure 1)

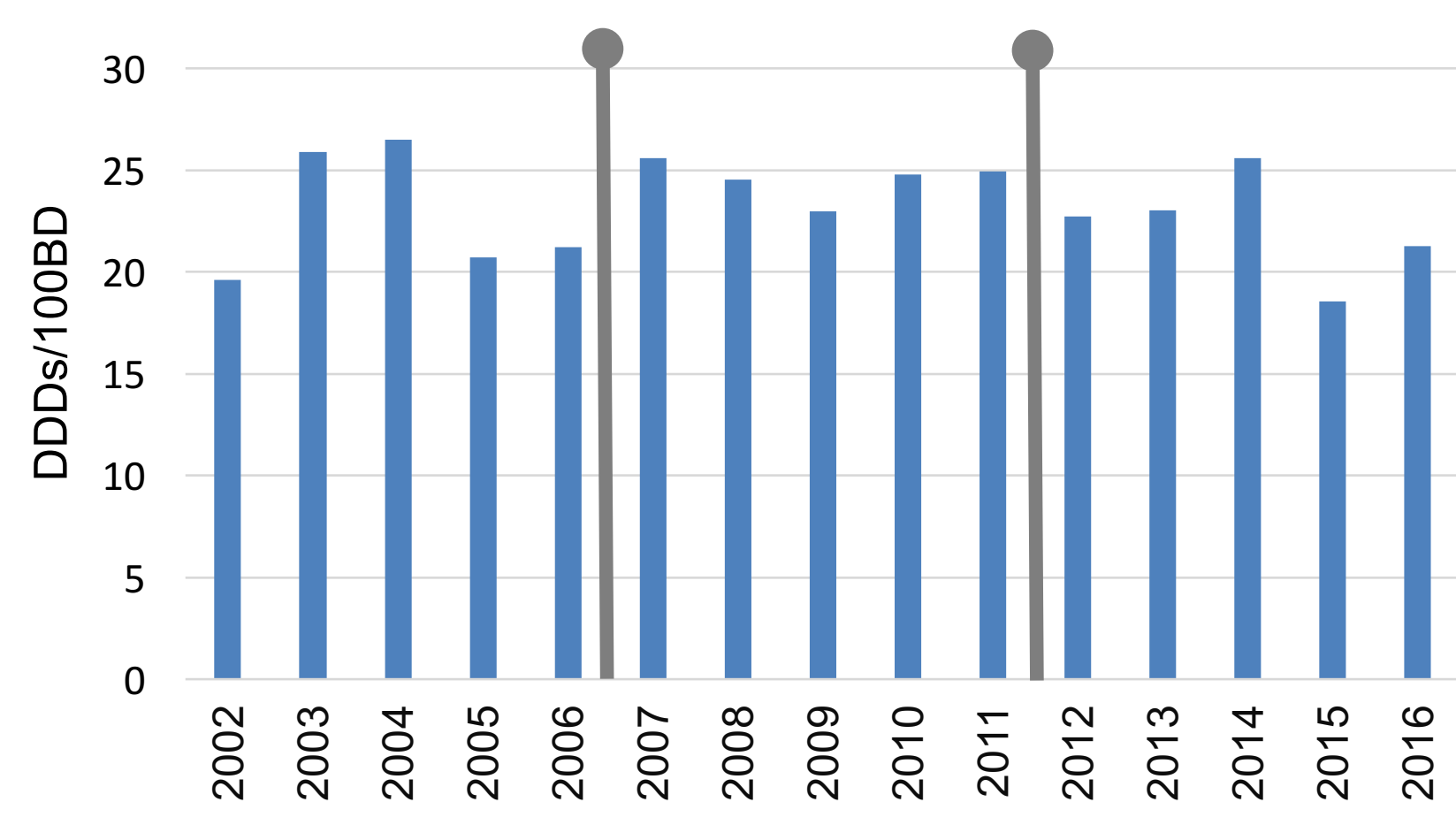

Figure 1: Total antimicrobial consumption in 3 study periods

Ampicillin (AMP) had the highest consumption among all antimicrobials and had a non-significant decrease especially during the third period (from 9.9 to 9.0DDD/100BD). Aminoglycosides (AMG) consumption followed ampicillin consumption and ranged between 3 and 4DDD/100BD.

During the first study period $3^{\text {rd }}$ and $4^{\text {th }}$ generation cephalosporins (3/4GC) were frequently used; however their consumption reduced from 4.3 to $1.8 \mathrm{DDD} / 100 \mathrm{BD}$ at the third time period. Carbapenem (CAR) consumption had no significant change (2.4-2.6DDD/100BD).

Glycopeptide (GLY) use showed an increase during the second period (from 2.3 to $3.1 \mathrm{DDD} / 100 \mathrm{BD}$ ) and then a reduction to $2.5 \mathrm{DDD} / 100 \mathrm{BD}$. The same trend was observed for fluoroquinolones (FQL), which had the lowest consumption: increased from 0.2 to $0.8 \mathrm{DDD} / 100 \mathrm{BD}$ and then reduced to $0.6 \mathrm{DDD} / 100 \mathrm{BD}$. (Figure 2)
Metronidazole consumption was constantly low (0.10.2DDD/100BD).

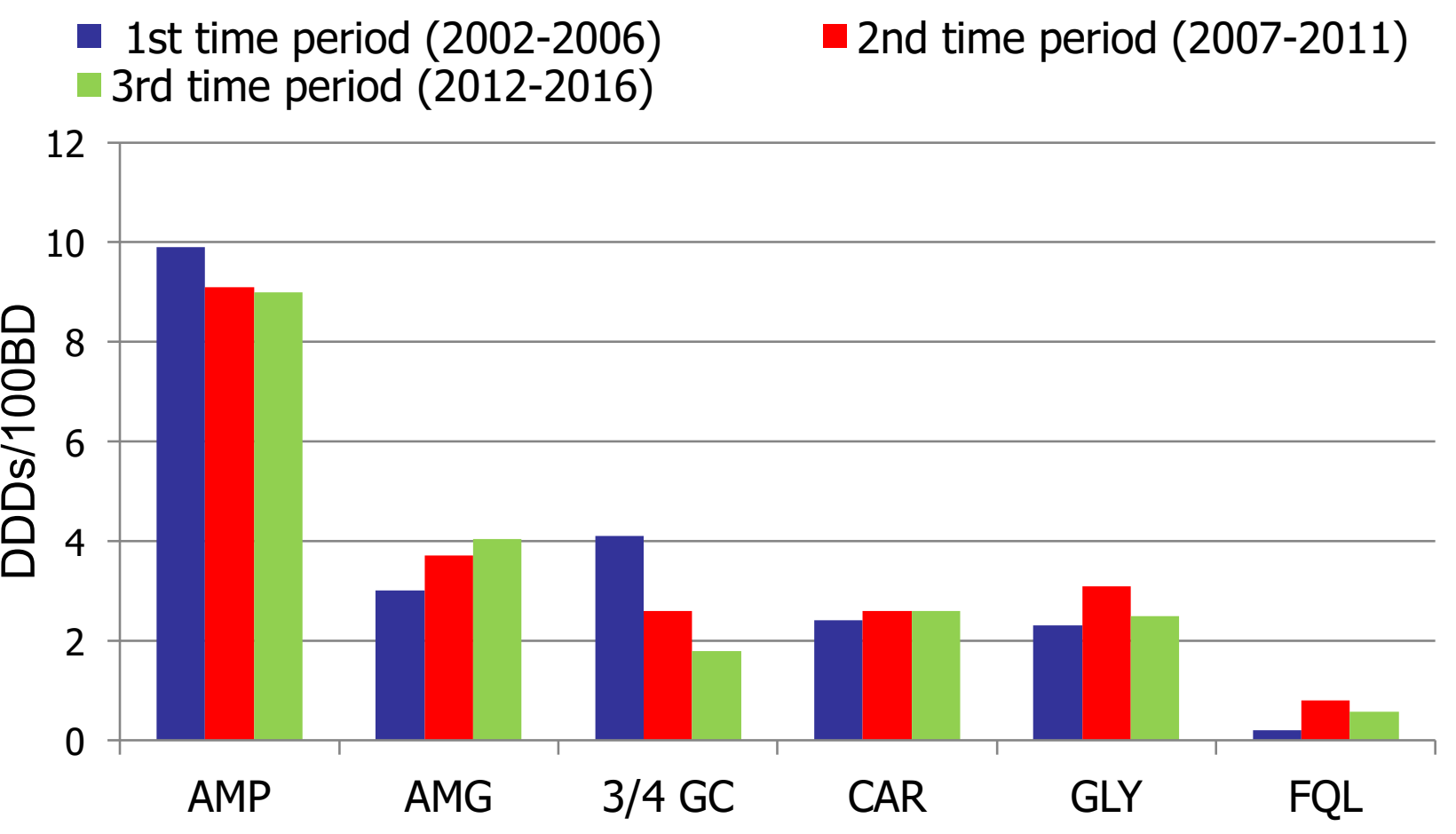

Figure 2: Antimicrobial consumption of commonly used antimicrobials

\section{Conclusion}

- Longitudinal analysis of antimicrobial consumption in NICU using the DDD methodology was feasible and provided analyzable data.

- Pattern and trends of antimicrobial consumption may be used for designing antimicrobial usage guidelines.

\section{References}

1. Cantey JB. et al, Reducing unnecessary antibiotic use in the neonatal intensive care unit (SCOUT): a prospective interrupted time-series study, Lancet Infect Dis., 2016 ;16(10):1178-1184.

2. Liem TB. et al., J Antimicrob Chemother, Variation in antibiotic use in neonatal intensive care units in the Netherlands, $2010 ; 65(6): 1270-5$.

3. WHO Collaborating Centre for Drug Statistics Methodology, Guidelines for ATC classification and DDD assignment 2018. Oslo, Norway, 2017

4. Monnet DL. ABC Calc - Antibiotic consumption calculator [Microsoft ${ }^{\circledR}$ Excel application]. Version 3.1. Copenhagen (Denmark): Statens Serum Institut; 2006 